\title{
Three-dimensional global fluid simulations of cylindrical magnetized plasmas
}

\author{
Naulin, Volker; Windisch, T.; Grulke, 0.
}

Published in:

Physics of Plasmas

Link to article, DOI:

$10.1063 / 1.2829603$

Publication date:

2008

Document Version

Publisher's PDF, also known as Version of record

Link back to DTU Orbit

Citation (APA):

Naulin, V., Windisch, T., \& Grulke, O. (2008). Three-dimensional global fluid simulations of cylindrical magnetized plasmas. Physics of Plasmas, 15(1), 012307. https://doi.org/10.1063/1.2829603

\section{General rights}

Copyright and moral rights for the publications made accessible in the public portal are retained by the authors and/or other copyright owners and it is a condition of accessing publications that users recognise and abide by the legal requirements associated with these rights.

- Users may download and print one copy of any publication from the public portal for the purpose of private study or research.

- You may not further distribute the material or use it for any profit-making activity or commercial gain

- You may freely distribute the URL identifying the publication in the public portal

If you believe that this document breaches copyright please contact us providing details, and we will remove access to the work immediately and investigate your claim. 


\title{
Three-dimensional global fluid simulations of cylindrical magnetized plasmas
}

\author{
V. Naulin, ${ }^{1}$ T. Windisch, ${ }^{2}$ and O. Grulke ${ }^{2}$ \\ ${ }^{1}$ Association EURATOM, Ris $\varnothing$ DTU, P.O. Box 49, DK-4000 Roskilde, Denmark \\ ${ }^{2}$ Max-Planck Institute for Plasma Physics, EURATOM Association, D-17491 Greifswald, Germany
}

(Received 7 August 2007; accepted 3 December 2007; published online 23 January 2008)

\begin{abstract}
Plasma dynamics in cylindrical geometry, with many well diagnosed experiments in operation worldwide, is of fundamental interest. These linear machines can provide an unique testing ground for direct and detailed comparisons of numerical simulations of nonlinear plasma dynamics with experiments. Thus, it is possible to assess the reproductive and predictive capabilities of plasma simulations in unprecedented detail. Here, three-dimensional global fluid simulations of a cylindrical magnetized plasma are presented. This plasma is characterized by the existence of spatially localized sources and sinks. The traditional scale separation paradigm is not applied in the simulation model to account for the important evolution of the background profiles due to the dynamics of turbulent fluctuations. Furthermore, the fluid modeling of sheath boundary conditions, which determine the plasma conditions, are an important ingredient to the code presented here. The linear properties of the model equations are studied and are shown to agree well with experimental observations of linear drift modes. The fully nonlinear simulations are characterized by turbulent fluctuations, which are dominated by low mode numbers in the large radial pressure gradient region. In the far plasma edge, the fluctuations display an intermittent character due to convection within radially extended spatiotemporal potential fluctuations. This paper reports on the model and general code results, while the detailed comparison to a specific experiment is left to a follow-up paper.

(C) 2008 American Institute of Physics. [DOI: 10.1063/1.2829603]
\end{abstract}

\section{INTRODUCTION}

Magnetized plasma in a cylindrical geometry is probably the most basic configuration for magnetized laboratory plasma devices. Typical examples are KIWI, ${ }^{1}$ MIRABELLE, ${ }^{2}$ LAPD (Large Plasma Device), ${ }^{3}$ the Auburn ALEXIS device (Auburn Linear Experiment for Instability Studies), ${ }^{4}$ SLPM (Santander Linear Plasma Machine) ${ }^{5}$ CSDX (Controlled Shear Decorrelation Experiment), ${ }^{6,7}$ and VINETA, ${ }^{8}$ to name only a few. The dynamics in these kind of plasma experiments is dominated by the presence of plasma sources and sinks. The sinks are usually localized at one axial end of the machine, where the magnetic field lines of the external magnetic field are in contact with material interfaces. The dynamical equilibrium between source and sink regions includes parallel plasma flow from the sources to the sinks. These flows are comparable in nature to similar parallel flows existing in the so-called scrape-off layer (SOL) of fusion devices, which is fueled by the transport crossing the last closed flux surface. In many respects the SOL determines the performance of a fusion plant and sets limits to its operation. While the basic processes of turbulence in the SOL have been identified within the last years, ${ }^{9}$ the detailed understanding of the interaction of the turbulence with the dynamical equilibrium is still incomplete. Characterization of the SOL, with the intent to later being able to influence its dynamics, is a prerequisite for controlled operation of an energy producing fusion power plant. However, detailed spatiotemporal data for the edge and SOL of fusion devices are difficult to obtain. Here, the smaller scale and-more importantly—lower temperature cylindrical experiments are in comparison generally extremely well diagnosed. Thus, the excellent quality of the detailed data available for profiles and fluctuations plus the relative simplicity of the cylindrical devices with respect to geometry makes them a natural testing ground for numerical simulations of global threedimensional (3D) plasma dynamics. Small scale linear devices, together with numerical simulations, can play an important role not only for the understanding and elucidation of basic plasma physics processes, but also for code validation efforts, thereby also contributing to a better understanding of SOL turbulence.

In this paper we present global fluid simulations of a cylindrical magnetized plasma with cold ions. We derive the governing equations for low frequency dynamics within the two-fluid equations without the usual Reynolds decomposition, separating background and fluctuations. The model reduces to the well known Hasegawa-Wakatani equations ${ }^{10}$ in the limit of small fluctuation amplitude and scale separation between background and fluctuations. It is, in the linear limit, equivalent to the models derived for linear analysis by Ellis et al. ${ }^{11}$ and more recently by Sosenko and co-workers, ${ }^{12}$ in which a background axial current is assumed.

The paper is organized as follows: In Sec. II we derive the basic equations in the framework of the drift approximation for the perpendicular velocities. We briefly discuss the linear dispersion relation in slab and cylindrical geometry in Sec. III, knowing that for cylindrical plasma devices there exists no static equilibrium. A specific point addressed, however, will be the radial variability of parameters in the cylindrical case. In Sec. IV we discuss parallel and perpendicular 
boundary conditions, which are a most important ingredient to the model. Section V is devoted to the numerical implementation and methods. We will especially discuss the importance of the nonlocal terms for the evolution of the system. In Sec. VI we present some results of our simulations and compare with experimental findings, before summarizing and discussing the results in Sec. VII.

\section{MODEL EQUATIONS}

While good progress has been made over the years explaining the observations in cylindrical plasma experiments by using two-dimensional (2D) simulations, most often detailed successes were limited to investigations of linear wave motion ${ }^{13}$ or to explaining specific basic physics mechanisms, as for example influencing and taming the weak turbulence observed in such devices. ${ }^{14}$ Reproducing steady state high poloidal wavenumbers or the full nonlinear behavior proves, however, extremely difficult and finally unsatisfactory, as reported recently in Ref. 6. This is thought to be due to the missing interaction between fluctuations and profiles in the 2D modeling, which leads to changes in the dynamics and statistics of fluctuations. ${ }^{15,16}$ Thus, our intent is to derive a minimal model for the global development of relatively cold, isothermal plasma in a cylindrical geometry, suited for numerical solution. We therefore use a fluid description of the plasma, starting with the continuity equations for electrons (e) and ions $(i)$,

$$
\frac{\partial n_{e, i}}{\partial t}+\nabla \cdot\left(n_{e, i} \vec{V}_{e, i}\right)=0
$$

$n_{e, i}$ being the electron and ion densities and $\vec{V}_{e, i}$ being the corresponding fluid velocities. Further, we use the ion mass $m_{i}=M$ and electron mass $m_{e}=m$, the pressure tensor $\mathbf{P}_{\mathbf{e}, \mathbf{i}}$ with $Z_{e}, i$ the charge number, which we will only have to use for the ions $Z=Z_{i}$. The force balance closes the isothermal system, where we note that neglecting electron temperature fluctuations leaves out one important mechanism to excite drift waves, ${ }^{17}$

$$
m_{e, i} n_{e, i} \frac{d \vec{V}_{e, i}}{d t}+\nabla \cdot \mathbf{P}_{\mathbf{e}, \mathbf{i}}-Z_{e, i} e n_{e, i}\left(\vec{E}+\frac{1}{c} \vec{V}_{e, i} \times \vec{B}\right)=\vec{F}_{e, i} .
$$

Besides the electric fields and the Lorentz force, we account for friction forces through $\vec{F}_{e, i}$. From Eq. (2), the drift velocities perpendicular to the ambient static magnetic field $\vec{B}$ $=B \hat{z}$ are determined, as the characteristic frequencies considered are much smaller than the cyclotron frequencies $\omega \ll \Omega_{i, e}$. We neglect electron inertia effects in the perpendicular motion so that the perpendicular drift velocity of the electrons is given by the $E \times B$ and diamagnetic drifts,

$$
\vec{V}_{\perp, e}=\frac{\vec{E} \times \hat{z}}{B}+\frac{1}{\Omega_{e} m n_{e}} \hat{z} \times \nabla P_{e} .
$$

For the cold ions $\left(T_{i} \approx 0\right)$, we keep the effects of ion inertia through the ion-polarization drift $\vec{V}_{p i}$,

$$
\vec{V}_{p i}=-\frac{M}{e Z B^{2}}\left[\partial_{t}+\left(\vec{V}_{E \times B}\right) \cdot \nabla_{\perp}-\mu_{i} \nabla_{\perp}^{2}\right] \nabla_{\perp} \phi .
$$

The ion polarization drift is relevant due to its nonvanishing divergence, i.e., the associated divergence of its current building up polarization charge, through which it enters the final equations, even though it is-in the usual drift scaling - one order of magnitude smaller when compared to the other drift velocities. The ion polarization drift also contains the effects of a centrifugal force on the ions, due to eventual rotation of the plasma. For low temperature, partially ionized plasmas collisions of the ions with the abundant neutral gas are relevant and lead to a polarizing ion current. Assuming the neutrals to be at rest we write for the friction force $\vec{F}_{i, n}=-M n_{i} \nu_{i, n} \vec{V}_{i}$. With the electric drift $\vec{V}_{E \times B}$ as the dominating velocity, we obtain perpendicular to the magnetic field

$$
\vec{V}_{P e d}=\frac{M}{e Z B^{2}} \nu_{i, n} \vec{E}_{\perp} .
$$

Quasineutrality $\left(\omega_{p i} \gg \Omega_{i}\right)$ demands zero divergence of the total current $\vec{J}=e n_{e}\left(\vec{V}_{e}-\vec{V}_{i}\right)$, which we write, separating as usual between parallel and perpendicular dynamics, as

$$
\nabla_{\perp} \cdot \vec{J}_{\perp}=-\nabla_{\|} \cdot \vec{J}_{\|}
$$

Note that $\rho_{s}^{2} \ll \lambda_{D e}^{2}$ is an equivalent formulation for $\omega_{p i} \gg \Omega_{i}$; thus, quasineutrality is necessary for being able to use the drift approximation for the perpendicular particle motions. Quasineutrality does not imply that there are no large scale fields in the plasma - it is merely a statement about the spatial and temporal scales under consideration. The importance of the nonlinear ion polarization drift becomes obvious as it gives the time dependence of the vorticity, where we use $n=n_{e}$,

$$
\begin{aligned}
\boldsymbol{\nabla}_{\perp} \cdot\left(\frac{n M}{Z B^{2}} \frac{d}{d t} \boldsymbol{\nabla}_{\perp} \phi\right)= & \mu_{i} \boldsymbol{\nabla}_{\perp} \cdot \frac{n M}{Z B^{2}} \nabla_{\perp}^{2} \boldsymbol{\nabla}_{\perp} \phi \\
& -\nabla_{\perp} \cdot \frac{n M}{Z B^{2}} \nu_{i} \nabla_{\perp} \phi+\nabla_{\|} e n(U-V) .
\end{aligned}
$$

Here we introduced $d / d t=\partial / \partial t+\vec{V}_{E \times B} \cdot \nabla$ for advection with the $E \times B$ velocity, and $U=V_{\|, i}$ and $V=V_{\|, e}$, respectively, for the parallel ion and electron velocities. For the parallel motion we note that the friction term in Eq. (2) can be split into two parts-one from collisions and one from thermal forces $\left(\vec{F}_{e}=\vec{F}_{C}+\vec{F}_{T}\right)$-with the collisional part originating mainly from electron-ion and electron-neutral collisions,

$$
\vec{F}_{C}=n e\left[\frac{\vec{J}_{\|}}{\sigma_{\|}}+\frac{\vec{J}_{\perp}}{\sigma_{\perp}}+\frac{m}{e} \nu_{e n} V \hat{z}\right],
$$

$\sigma$ being the conductivity $\sigma_{\|} \approx 2 \sigma_{\perp}=2 n e^{2} / m \tau_{e}$. The thermal forces are neglected here, as we not only consider an isothermal plasma, but in addition parallel thermalization is a fast process, so that parallel temperature gradients can be neglected. In the electrostatic approximation $\vec{E}_{\|}=-\nabla_{\|} \phi$, we write 


$$
\frac{d}{d t} V=\frac{e}{m} \boldsymbol{\nabla}_{\|} \phi-\frac{T}{m} \nabla_{\|} \ln n-\nu_{e i}(V-U)-\nu_{e n} V
$$

and for the cold ions

$$
\frac{d}{d t} U=-\frac{e Z}{M} \boldsymbol{\nabla}_{\|} \phi-\frac{m}{M} \nu_{e i}(U-V)-\nu_{i n} U .
$$

The parallel velocities are here not replaced by the parallel current, as is usually done when considering fluctuations only. Dimensionless variables are introduced by measuring length and time with values typical for electron drift dynamics, namely, the ion gyrofrequency $\Omega_{i}=e Z B / M$, the sound speed $c_{s}^{2}=T_{e} / M$, and the ion Larmor radius at electron temperature $\rho_{s}=c_{s} / \Omega_{i}$,

$$
\breve{l}=\frac{l}{\rho_{s}}, \quad \breve{t}=t \Omega_{i}, \quad \breve{V}=\frac{V}{c_{s}},
$$

and choose arbitrary reference levels for electron temperature, density, and magnetic field,

$$
\breve{\phi}=\frac{e \phi}{T_{e, 0}}, \quad \breve{n}=\frac{n}{n_{0}}, \quad \breve{B}=\frac{B}{B_{0}} .
$$

We introduce

$$
\nu_{e}=\frac{\nu_{e n}}{\Omega_{i}}, \quad \nu_{i}=\frac{\nu_{i n}}{\Omega_{i}}, \quad \text { and } \quad \nu_{\|}=\frac{\nu_{e i}}{\Omega_{i}} .
$$

Finally, we write $\boldsymbol{\nabla}=\boldsymbol{\nabla}_{\perp}$, measure masses relative to the

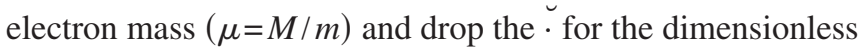
quantities,

$$
\begin{aligned}
& Z \nabla N \cdot \frac{d}{d t} \boldsymbol{\nabla} \phi+Z \frac{d}{d t} \Omega=\nabla N \cdot\left(\mu_{\Omega} \nabla \Omega-Z \nu_{i} \nabla \phi\right) \\
& -Z \nu_{i} \Omega+\nabla_{\|}(U-V) \\
& +(U-V) \nabla_{\|} N+\mu_{\Omega} \nabla^{2} \Omega \text {, } \\
& d_{t} N=-\left(V \nabla_{\|} N+\nabla_{\|} V\right)+\mu_{n}\left[\nabla^{2} N+(\nabla N)^{2}\right], \\
& d_{t} U=-Z \nabla_{\|} \phi-\nu_{i} U-\frac{\nu_{\|}}{\mu}(U-V), \\
& d_{t} V=\mu \nabla_{\|}(\phi-N)-\nu_{e} V-\nu_{\|}(V-U) .
\end{aligned}
$$

We further used $N=\ln n$, introduced the vorticity $w=\nabla^{2} \phi$, and added viscous and diffusive terms $\sim \mu_{\Omega}$ and $\mu_{n}$ to the vorticity and density equations, respectively. This set of equations has been used in Ref. 16 to investigate the statistics of fluctuations in a tokamak at the transition from closed to open magnetic field lines and lately with constant coefficients and for immobile ions $(U=0)$ in Ref. 18. Note that these equations reduce to the ones used by Wakatani et al. ${ }^{19}$ in the case of vanishing parallel ion-velocity and when only fluctuations are considered. It is further an interesting fact that the final equations can be completely written in terms of the logarithm of the density, signifying the fact that the density cannot take negative values.

\section{LINEAR PROPERTIES}

Here we discuss briefly the linear properties of the derived system. For simplicity we exclude the parallel ionvelocity from our considerations and consider the ions as providing a fixed background.

\section{A. Slab geometry}

In slab geometry, we linearize around a constant background density with a fixed and constant density gradient as this is the usual starting point for the analysis of the experimental situation. This background is by no means special, its only "equilibrium" properties are that in the absence of diffusion any radial density profile would indeed be a solution to the system proposed above. To further reflect the situation commonly present in experiments we additionally consider a constant background electron drift $V_{0}$, which corresponds to an electron current through the discharge as appropriate for many devices. It is assumed that electron inertia can be neglected, ions are at rest $(U=0)$, and that the system can be reduced to effectively two dimensions by introducing one principal wavelength $k_{\|}$, which dominates the axial structure. The fields are decomposed in the perpendicular direction into Fourier components in the standard way; i.e., $A_{k_{\|}}$ $=\sum_{\vec{k}} A_{\vec{k}, k_{\|}} \exp -i \omega t+i \vec{k} \cdot \vec{x}$ with $\vec{k}=\left(k_{x}, k_{y}\right)$ being the perpendicular wave vector. With $\tilde{\tau}=\mu / \nu_{\|}$we obtain using the diamagnetic frequency $\omega^{*}=k_{y} / L_{n}$

$$
\begin{aligned}
& i Z \omega k^{2} \phi=\mu k^{4} \phi+\widetilde{\tau} k_{\|}^{2}(\phi-n)-i V_{0} k_{\|} n+\nu_{i} Z k^{2} \phi, \\
& -i \omega n=-i \omega^{*} \phi+\widetilde{\tau} k_{\|}^{2}(\phi-n)-i V_{0} k_{\|} n .
\end{aligned}
$$

Introducing $\tau=\left(1 / \nu_{\|}\right)(M / m) k_{\|}^{2}=\tilde{\tau} k_{\|}^{2}, \Omega_{1}=V_{0} k_{\|}$, and $b=k^{2}$, the quadratic expression for the dispersion relation follows:

$$
\begin{aligned}
& Z b \omega^{2}+\omega\left[i \tau(1+Z b)+i b\left(Z \nu_{i}+\mu b\right)-Z \Omega_{1} b\right] \\
& \quad-\tau\left[i \omega^{*}+b\left(Z \nu_{i}+\mu b\right)\right]+\Omega_{1}\left[\omega^{*}-i b\left(Z \nu_{i}+\mu b\right)\right]=0 .
\end{aligned}
$$


Evaluating Eq. (14) close to adiabaticity, i.e., $\tau \rightarrow \infty$, we find the usual drift-wave branch of the dispersion relation, with the real frequency

$$
\omega_{r}=\frac{\omega^{*}}{1+Z k^{2}}
$$

and the growth rate

$$
\begin{aligned}
\omega_{i}= & \frac{\omega^{\star}}{\tau\left(1+Z k^{2}\right)}\left[\frac{Z k^{2} \omega^{\star}}{\left(1+Z k^{2}\right)^{2}}+Z \Omega_{1}\right] \\
& -\frac{k^{2}}{1+Z k^{2}}\left(Z \nu_{i}+\mu k^{2}\right) .
\end{aligned}
$$

This recovers the drift dissipative instability, which vanishes as $\tau$ goes to infinity, as with that the electrons are able to instantaneously account for pressure and potential fluctuations by rapid motion along the field lines. For an instability to occur, there must also be a background density gradient $\left(\omega^{\star}\right)$ to provide the free energy for the growth of the fluctuations. A second mechanism for instability lies in the drifting electrons $\left(\Omega_{1}\right)$, as the drift affects the phase relation between density and potential fluctuations. Note that the sign of the phase shift, and by this the instability, is dependent on the sign of $V_{0}$ in relation to $\omega^{\star}$. The same dispersion relation was derived by Ellis and co-workers ${ }^{11}$ [note that in their formula (10b) a factor $b$ is missing from the collisional instability rate].

\section{B. Cylindrical geometry}

While the linear dispersion in a doubly periodic setup is an easy exercise, it is more difficult to solve the dispersion relation in a cylindrical setup, where we have to find the mode structure fitting the exact profiles and obeying boundary conditions. The radial boundary conditions are generally not well known, as they depend partly on properties of the vessel wall. Therefore, we here use simplified boundary conditions. For the density, as well as for the potential, we introduce background profiles

$$
n=n_{0}(r)(1+\tilde{n})
$$

and

$$
\phi=\phi_{0}(r)+\tilde{\phi}
$$

and consequently,

$$
\Omega=\Omega_{0}(r)+\tilde{\Omega} .
$$

We further introduce the following abbreviations:

$$
\begin{aligned}
& \kappa:=\partial_{r} \ln n_{0}, \\
& V_{p}:=\partial_{r} \phi_{0}, \\
& S_{p}:=\partial_{r} \Omega_{0}=\partial_{r}\left(\frac{1}{r} \partial_{r}+\partial_{r r}\right) \phi_{0} .
\end{aligned}
$$

We have, then, dropping the tilde and considering singly charged ions $Z=1$,

$$
\begin{aligned}
\partial_{t} \Omega-V_{p} \frac{1}{r} \partial_{\theta} \Omega= & -\nu_{i} \Omega-\nabla_{\|} V-V_{0} \nabla_{\|} n-\kappa\left(\frac{\partial}{\partial t}+\nu_{i}\right) \partial_{r} \phi \\
& +\kappa V_{p} \frac{1}{r} \partial_{\theta} \partial_{r} \phi-\nu_{i} V_{p} \partial_{r} n-S_{p} \frac{1}{r} \partial_{\theta} \phi, \quad(17)
\end{aligned}
$$

$$
\begin{aligned}
& \partial_{t} n-V_{p} \frac{1}{r} \partial_{\theta} n=-\kappa \frac{1}{r} \partial_{\theta} \phi-\left(V_{0} \nabla_{\|} n+\nabla_{\|} V\right), \\
& \partial_{t} V-V_{p} \frac{1}{r} \partial_{\theta} V=\mu \nabla_{\|}(\phi-N)-\left(\nu_{e}+\nu_{\|}\right)\left(V+V_{0}\right) .
\end{aligned}
$$

If we neglect electron inertia, and use poloidal periodicity $\sim \exp (i \tilde{m} \theta)$, with $\tilde{m}$ being the poloidal mode number (to be distinguished from electron mass $m$ ), we can solve for a static relationship determining the parallel electron velocity from density and potential using Eq. (19),

$$
V-\frac{V_{p} \frac{1}{r} \partial_{\theta} V}{\nu_{e}+\nu_{\|}}=\frac{\mu \nabla_{\|}(\phi-n)}{\nu_{e}+\nu_{\|}}-V_{0} .
$$

Now we make the following ansatz for the fluctuating quantities $V, \Omega, n, \phi \sim \exp \left(-i \omega t+i k_{\|} z+i \tilde{m} \theta\right)$, and find for $\tilde{m}>0$, as $V_{0}$ is a function of radius only:

$$
\left(1-\frac{i \tilde{m} V_{p}}{r\left(\nu_{e}+\nu_{\|}\right)}\right) V=\frac{\mu \nabla_{\|}(\phi-n)}{\nu_{e}+\nu_{\|}} .
$$

Defining the operators

$$
P:=\frac{\mu k_{\|}^{2}}{\left(\nu_{e}+\nu_{\|}\right)-\frac{i \tilde{m} V_{p}}{r}}
$$

and $\Omega_{1}:=V_{0} k_{\|}$results in

$$
-i \widetilde{\omega} n+i \frac{\tilde{m}}{r} \kappa(r) \phi-P(\phi-n)+i \Omega_{1} n=0,
$$

from which the phase relation between density and potential fluctuations follows:

$$
n=\frac{\omega^{*}+i P}{\tilde{\omega}-\Omega_{1}+i P} \phi
$$

with the diamagnetic frequency $\omega^{*}=-(\tilde{m} / r) \kappa(r)$ and the Doppler shifted frequency $\widetilde{\omega}=\omega+\tilde{m} V_{p} / r$. Finally, we obtain the following eigenproblem:

$$
\partial_{r r} \phi+\left(\frac{1}{r}+\kappa-i \nu_{i} R_{D}\right) \partial_{r} \phi+\left[Q(r)-\frac{\tilde{m}^{2}}{r^{2}}\right] \phi=0
$$

with

$$
Q(r):=\frac{1}{\widetilde{\omega}+i \nu_{i}}\left[\omega^{*}-\frac{\widetilde{\omega}\left(\omega^{*}+i P\right)}{\tilde{\omega}-\Omega_{1}+i P}-\frac{\tilde{m}}{r} S_{p}\right],
$$

plus the rotational drag term 


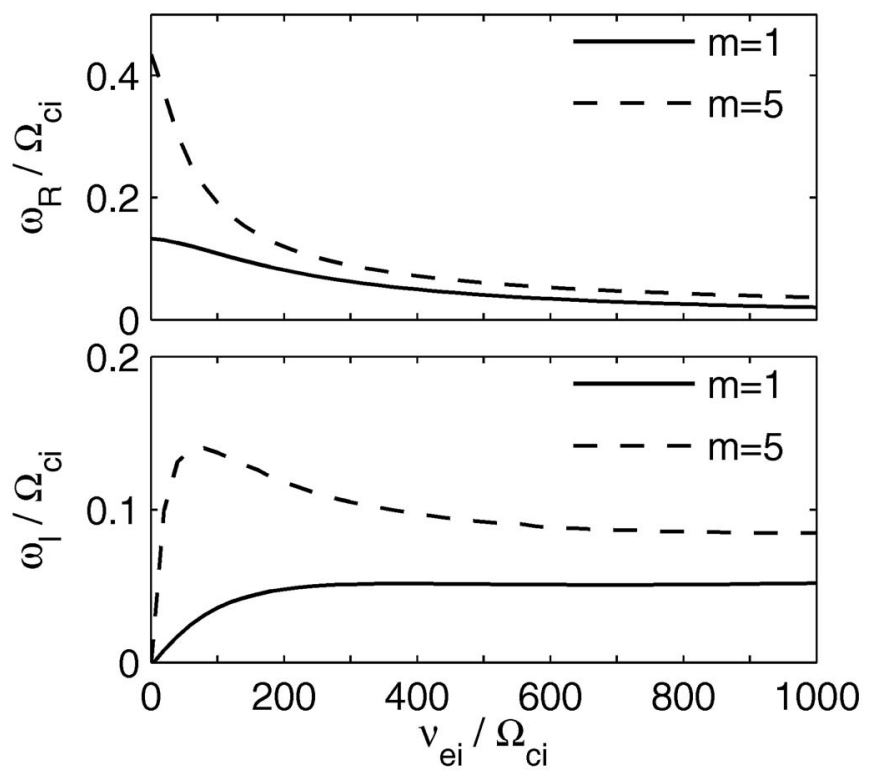

FIG. 1. Results of the linear drift wave dispersion calculations in terms of the frequency $\omega_{r}$ and growth rate $\omega_{i}$ for variations of the electron-ion collision frequency. All frequencies are normalized to the ion gyrofrequency $\Omega_{i}$. Shown are the results for the $\tilde{m}=1$ (dashed line) and the $\tilde{m}=5 \mathrm{drift}$ mode (solid line).

$$
R_{D}=\frac{1}{\widetilde{\omega}+i \nu_{i}} V_{p}
$$

The resulting system is solved numerically using a shooting algorithm and scanning the $\left(\omega_{r}, \omega_{i}\right)$ space looking for solutions without a node in the radial domain. It should be noticed that details of the dispersion depend on the extent of the numerical domain, as it is difficult for the shooting algorithm to converge to the same solution if the radial boundary point is moved outwards. However, the principal results can be summarized as follows. If the circumference at the maximum gradient radius is large, measured in $\rho_{s}$, the most unstable mode can be well estimated from the linear slab dispersion relation. For smaller circumference, up to around a mode number of $\tilde{m} \leq 4$, boundary effects are important. The dependence of the drift wave frequency and growth rate on the parallel electron-ion collision frequency has been studied intensely for low mode numbers $\widetilde{m} \leq 5$ and $Z=1,{ }^{20}$ and is explicitly depicted in Fig. 1. Shown are the evolution of $\omega_{r} / \Omega_{i}$ and $\omega_{i} / \Omega_{i}$ with collision frequency $\nu_{e i} / \Omega_{i}$ for the $\tilde{m}=1$ and $\tilde{m}=5$ drift wave modes. The drift mode frequency $\omega_{r}$ decreases with increasing collision frequency. This behavior is most pronounced for the low mode numbers with a drop in frequency of one order of magnitude for the $\widetilde{m}=1$ mode. In the collisionless limit the frequency approaches the Hasegawa-Mima limit frequency. The linear growth rate for the $\widetilde{m}=5$ mode increases with collision frequency. The same finding holds true for all modes with mode numbers $\tilde{m}>1$. The monotonic behavior only breaks for the $\tilde{m}=1$ mode, for which the growth rate peaks at a collision frequency of $\nu \approx 75$.

In high-density, low-temperature laboratory experiments, the plasma has usually strong radial gradients, which alter
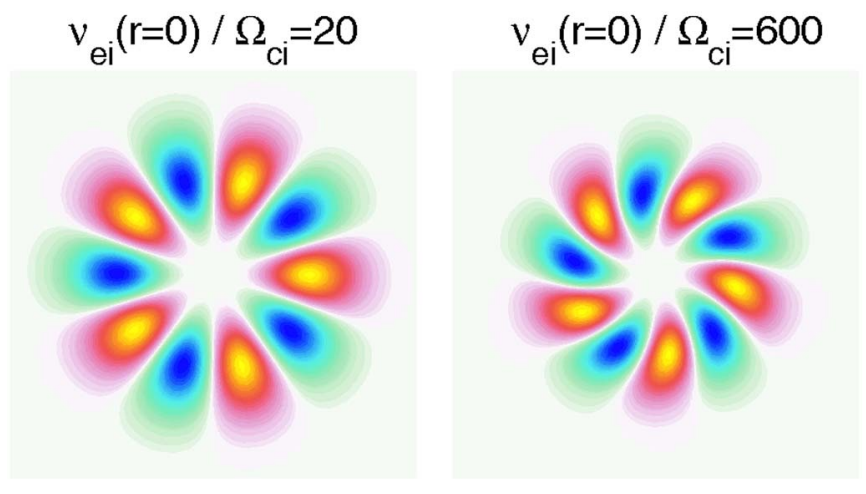

FIG. 2. (Color online) Spatial mode pattern for an $\tilde{m}=5$ mode in the azimuthal plane for small peak collision frequency (left) and high peak collision frequency (right). The plasma density of the mode is shown color coded.

radially the Coulomb collision frequency. The spatial mode structure in the azimuthal plane for an assumed radial Gaussian collision profile is shown in Fig. 2. Two cases are distinguished: Small peak collision frequency in the plasma center $\left(\nu_{e i} / \Omega_{i}=20\right)$ and large peak collision frequency $\left(\nu_{e i} / \Omega_{i}\right.$ $=600$ ). In the low collisionality case the drift wave eigenmodes have a purely radial dependence, whereas in the high collisionality case the mode develops an azimuthally bent eigenmode structure in response to the radial variation of the mode frequency. It was shown that the bending of the mode structure is in excellent agreement with experimental results. ${ }^{13}$ This finding demonstrates the strong coupling between the parallel electron dynamics and the perpendicular drift mode frequency and is not directly indicative of a shear flow winding up an otherwise straight radial mode structure.

\section{BOUNDARY CONDITIONS}

Boundary conditions for turbulence simulations on bounded domains are not trivial. For plasmas, no equivalent to the no-slip boundary condition of fluid dynamics exists and moreover, for the situation of magnetic field lines parallel to the walls, considering complicated atomic physics would be appropriate for detailed modeling. To achieve realistic boundary values for the electromagnetic fields, one would have to model currents in the metal casing that surrounds the plasmas as well as the outer circuitry. As we are interested in reproducing the most robust dynamics of the plasma system with the least complicated boundary conditions we try to fix as simple as possible boundary conditions.

At one axial end $(\mathrm{z}=0)$ of the cylinder under consideration we prescribe a plasma density source and do not consider sheath boundaries there. Thus, at $z=0$, all dynamical quantities are assumed to have zero parallel derivative. The source density is prescribed to be of Gaussian shape with a fixed parallel decay length,

$$
S=\exp \left(-r^{2} \kappa_{n}^{2}\right) \exp \left(-z^{2}\right),
$$

and $S / n$ is added to the right-hand side of Eq. (9). At the opposite end of the cylinder $\left(z=L_{z}\right)$, the anomalous density flux $n v_{\|}$is assumed to have zero axial derivative, allowing plasma to leave the device along magnetic field lines, while 
TABLE I. Typical parameters for three selected experiments.

\begin{tabular}{lccc}
\hline \hline Name & KIWI (Ref. 26) & MIRABELLE (Ref. 12) & VINETA \\
\hline$n_{0}$ & {$[1-7] \times 10^{10} \mathrm{~cm}^{-3}$} & {$[1-7] \times 10^{10} \mathrm{~cm}^{-3}$} & $1 \times 10^{11} \mathrm{~cm}^{-3}$ \\
$T_{e}, \mathrm{eV}$ & 2 & 2.5 & 3 \\
$T_{i}, \mathrm{eV}$ & $0.03 \mathrm{eV}$ & 0.05 & 0.1 \\
$B, \mathrm{~T}$ & 0.07 & 0.04 & 0.06 \\
$r_{\text {plasma, }} \mathrm{cm}$ & $4-7$ & 14 & 10 \\
$r_{\text {profile, }} \mathrm{cm}$ & 2.5 & $7-11$ & 7 \\
$l_{\|}, \mathrm{cm}$ & 150 & 140 & 450 \\
$v_{\|}$ & {$[0.1-0.2] v_{\text {th }, e}$} & $?$ & 0 \\
$r_{\text {vessel, }} \mathrm{cm}$ & 30 & 30 & 40 \\
\hline \hline
\end{tabular}

potential and velocity boundary conditions are determined by simple Bohm sheath boundary conditions. A rather complete discussion of sheath boundary conditions can be found in the book by Stangeby, ${ }^{21}$ and more condensed in the article by Post et al. ${ }^{22}$ Usually, the Bohm condition determines the current into the sheath being regulated by the sheath entry potential,

$$
j=e n\left[c_{s}-\frac{1}{\sqrt{2 \pi}} \sqrt{\frac{T_{e}}{m}} \exp \left(-\frac{e \phi_{\text {sheath }}}{T_{e}}\right)\right],
$$

or in dimensionless quantities for the parallel electron velocity at the sheath entry

$$
V_{\mid \partial}=-\frac{1}{\sqrt{2 \pi}} \sqrt{\mu} \exp \left(-\phi_{\mid \partial}\right) .
$$

Thus, the potential regulates the outflow of the electrons, while for the parallel ion-velocity, we assume that at the sheath the ions reach the sound velocity; that is,

$$
U_{\mid \partial}=1 \text {. }
$$

Clearly, in steady state, on average, $\left\langle V_{\mid \partial}\right\rangle=1$ is reached and the current decays to be zero on average (unless a current is specified via a background electron drift), with $\langle\cdot\rangle$ indicating a temporal average over a long enough time. We further assume that the plasma density decreases radially to a finite level $N_{b d}$ at $r=a$, which is determined by the source shape. The surrounding plasma casing is taken to be an ideal conductor which fixes the potential to be zero at the walls, defining the reference potential to be the one that results in zero current into the sheath, which is necessary to keep the radial and axial boundary conditions consistent. The boundary condition for the vorticity is consequentially zero as well. Parallel ion and electron velocities are assumed to have zero radial gradient at $r=a$. One should note that in this setup the potential is determined on each plane perpendicular to the magnetic field independently. This a pronounced consequence; namely, that using periodic boundary conditions in the radial direction, would demand solving Poisson equation in three dimensions, as a finite average polarization charge (vorticity) is in the kernel of the Laplace operator for these boundary conditions. The parallel variation of the potential would thus get lost using the 2D Poisson equation to determine the potential. Fixing a reference potential on the wall removes that
TABLE II. Input parameters for the simulations presented.

\begin{tabular}{lc}
\hline \hline Name & Value \\
\hline$z_{\max }$ & 150 \\
$x_{\max }$ & 30 \\
$\nu_{\|}=\nu_{e i} / \Omega_{i}$ & 4.81 \\
$\nu_{i}=\nu_{i n} / \Omega_{i}$ & 0.0553 \\
$\nu_{e}=\nu_{e n} / \Omega_{i}$ & 10.8 \\
$\mu=0.3\left(T_{i} / T_{e}\right)\left(\nu_{i} / \Omega_{i}\right)$ & 0.0005 \\
$\kappa_{n}$ & 1.3 \\
Length unit $\rho_{s}$ & $1.86 \mathrm{~cm}$ \\
Time unit $1 / \Omega_{i}$ & $5 \mu \mathrm{s}$ \\
\hline \hline
\end{tabular}
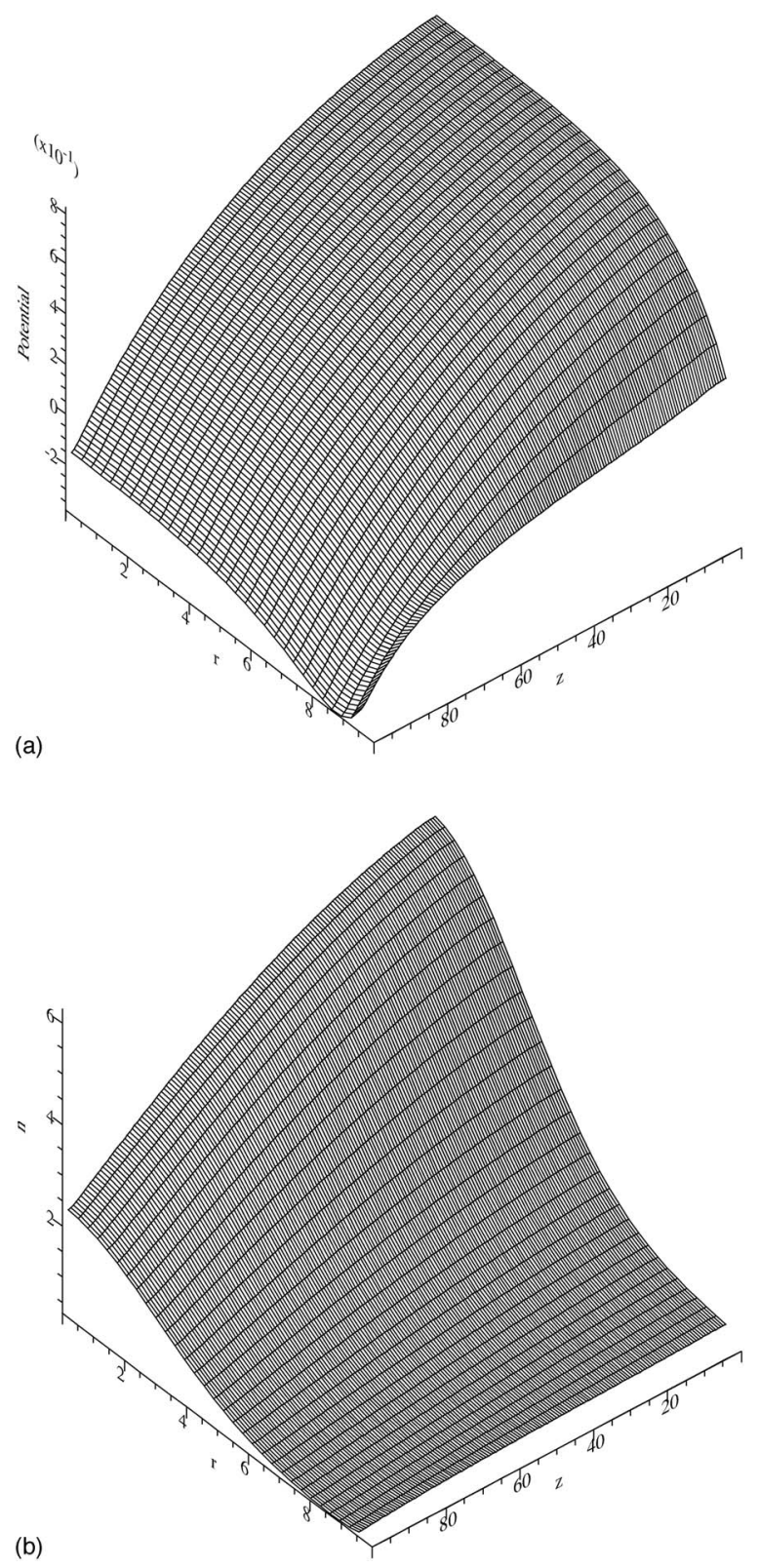

FIG. 3. Snapshot of dimensionless plasma potential (top) and density (bottom) at zero poloidal angle in the steady state with low amplitude fluctuations. The plasma is charged up positively, as electrons get lost to the sheath initially. In this state parallel pressure gradient and electric field balance. In situations with turbulence, the plasma density profile is strongly disturbed by radial anomalous transport. 

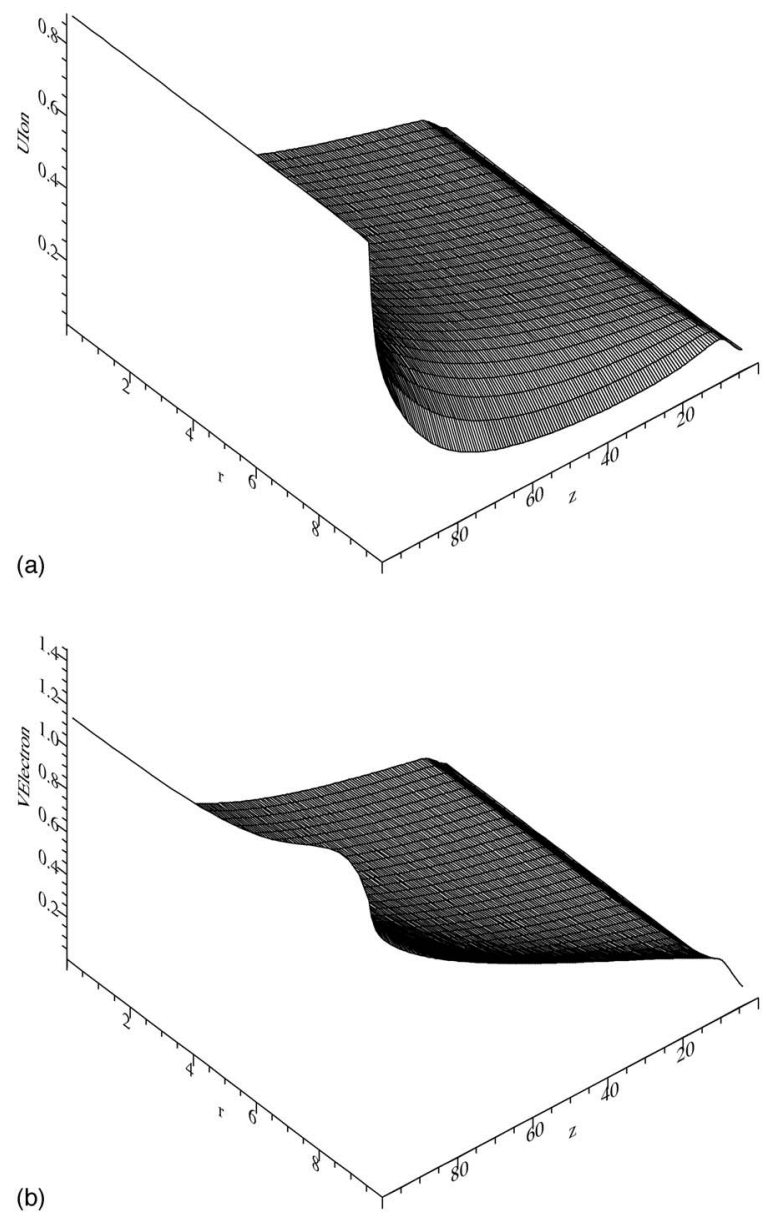

FIG. 4. Snapshots of ion (top) and electron (bottom) velocities normalized to the ion sound speed at zero poloidal angle in the steady state with low amplitude fluctuations. Both ions and electrons accelerate towards the sheath entry. Both velocities develop self-consistently and balance on average in steady state as the net current falls to zero average.

problem, and the numerical effort for the system can be kept down to solving 2D Poisson problems only, with beneficial consequences for the numerical complexity of the code and its implementation in parallel.

\section{NUMERICAL IMPLEMENTATION}

The model equations are solved using finite difference discretizations. The density as a strictly positive definite quantity is implemented in terms of its logarithm, thus avoiding numerical problems of the density falling below zero in cases of steep gradients forming in the simulations, which lead to numerical oscillations (also known as ringing or overshooting). The parallel derivatives are implemented as fourth-order centered finite differences. All nonlinear terms are formulated in terms of Poisson brackets

$$
\{a, b\}=\frac{1}{r} \partial_{r} a \partial_{\phi} b-\partial_{r} b \partial_{\phi} a
$$

and the Arakawa discretization ${ }^{23}$ is used, which reflects the conservation properties of the Poisson bracket structure.

The coordinate singularity of the cylindrical grid with spacing $(\Delta r, \Delta \phi, \Delta z)$ at $r=0$ is avoided by using a staggered

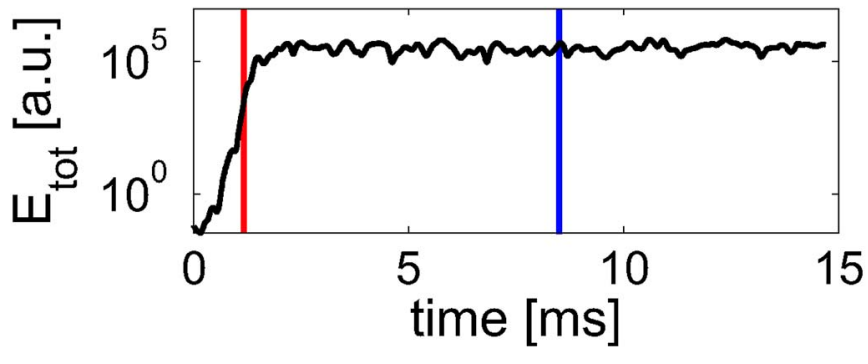

FIG. 5. (Color online) Temporal evolution of the total energy as semilogarithmic representation. The vertical bars indicate the time instants for the spatiotemporal plots of plasma density fluctuations, Fig. 8.

grid with allocation points at $x_{i}=i \Delta r+0.5 \Delta r$. Boundary conditions are implemented by using ghost-points: One ghostpoint radially and two ghost-points in the $z$ direction. The numerical resolution is $(n x, n y, n z)$ points in the corresponding directions. To further avoid unreasonably short timesteps as a consequence of satisfying the Courant-FriedrichsLevy condition for $r \Delta \phi \rightarrow 0$ as $r \rightarrow 0$, we keep only modes with $m<0.25 n y r / r_{0}$. Modes with larger mode numbers are removed in the implicit part of the time-step. The timestepping scheme is a third-order embedded gear method, also know as a stiffly stable scheme, following the layout by Karniadakis. ${ }^{24}$ The scheme uses operator splitting, treating all terms on a explicit basis besides the dissipative ones, which are stepped forward in time using an implicit scheme. The implicit scheme demands solving a Helmholtz equation, which is done using fast Fourier transform in the periodic poloidal direction and solving the remaining tridiagonal matrix by a direct method. The same solver is used to determine the potential from the vorticity, where it is appropriate to note that the first term of the left-hand side of the vorticity equation (8) is evaluated in an iterative manner. This combination of spatial and temporal discretization has been tested in Naulin and Nielsen ${ }^{25}$ and compared favorably with spectral as well as with essentially nonoscillatory schemes.

All simulations are initialized by using a constant background density and zero parallel velocities. The initial timestep then needs to be about a factor of 10 shorter compared to the time-step during the developed simulations due to large gradients developing in the initial conditions at the sheath entry. Therefore, the simulations are usually started up with a short time-step running to one or two sound transit times $t_{\text {trans }}=L_{\|} / c_{s}$. The simulation is then stopped and subsequently restarted from that output file for different parameters and importantly with a larger time-step.

\section{SIMULATION RESULTS}

The 3D simulations are run for a variety of parameters in the range indicated by three typical cylindrical experiments, whose relevant parameters can be found in Table I. Detailed parametric scans as well as comparison to a specific experiment will be reported in a follow-up paper.

We report here on simulations performed for VINETA parameters using for the numerical simulations a grid of 32 $\times 64 \times 16$ points in radial, azimuthal, and parallel directions, respectively. In the following, the most important properties 

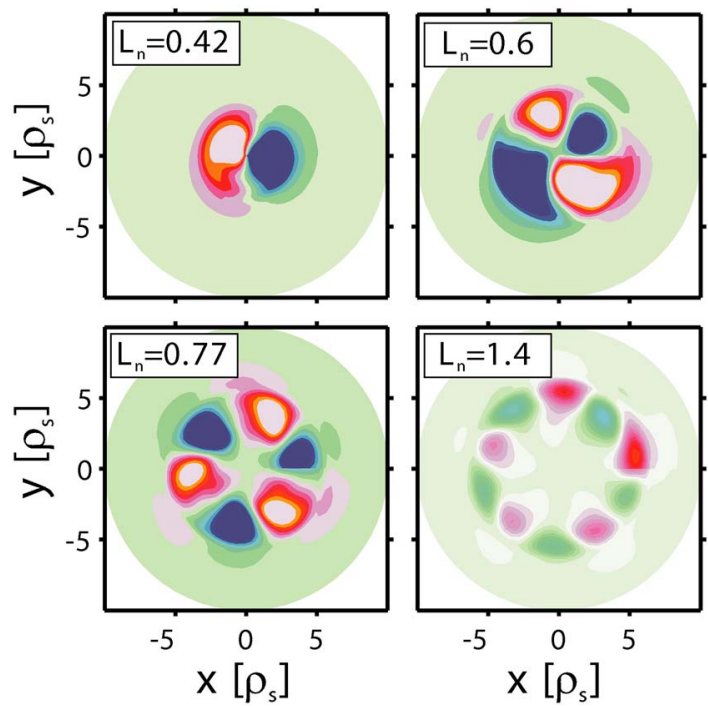

0.05

FIG. 6. (Color online) Mode structure for increasingly wider source profiles, showing the transition to higher mode numbers.

of a simulation run are reported. Input parameters correspond to a linearly magnetized high-density, low-temperature laboratory experiment, in which the peak plasma collisionality is high compared to the ion gyrofrequency. The list of relevant input parameters is compiled in Table II.

Initially, the density in the numerical simulation volume is constant and parallel velocities increase axially from 0 to 1 at the sheath entry. The plasma density increases in the source region close to the axially first grid-point. While the parallel pressure gradient pushes the electrons out and toward the end plate, the plasma charges up positively in the plasma source region. This in turn slows down the parallel electron motion until a steady state is reached by which the plasma is expanding roughly with ion sound speed toward the target plate. In this phase, steep gradients appear transiently at the sheath and fast oscillations occur in the simulation, which are due to the fact that the parallel force acting on the electrons is not yet balanced. In this case, the subsystem

$$
\partial_{t} \Omega=-\nabla_{\|} V \text { and } \partial_{t} V=\mu \boldsymbol{\nabla}_{\|} \phi
$$

dominates the dynamics, with high frequencies present due to the large ratio of ion over electron inertia. Consequently, the time-step has to be very small until a state is reached where the parallel force on the electrons is small.

After some ion sound transit times $t_{\text {trans }}=L_{\|} / c_{s}$, the simulation has reached this quieter phase. The plasma column is filled with plasma and instead of being dominated by the parallel processes leading to this state, the plasma dynamics is then governed by instabilities and turbulence that develop on the background given by the dynamical equilibrium between source and sink. The dynamical equilibrium is characterized by a positively charged up plasma column in the source region, as initially electrons are pushed out from that region, as seen in Fig. 3, where a snapshot of the potential and density at zero poloidal angle is shown. On average, the parallel gradient of the potential will compensate the parallel gradient of the logarithmic density, leading to a (nearly) vanishing parallel force on the electrons. In the same figure, the density shows a significant decay from the source at $z=0$ to the sheath. Ion and electron velocity profiles are increasing axially from zero toward ion sound speed $c_{s}$, as can be seen

from Fig. 4. The short time scales involved in the electron velocity make this quantity prone to numerical distortions. The electron parallel velocity also shows large scale parallel structure in the region where the later instability evolves. It should be noted that while this leads to fluctuations in the parallel current, the time-averaged current through the cylinder has decayed to zero in the steady state (unless a net voltage and a net electron drift is kept up between source and sink region).

The dynamics in the perpendicular direction evolves on a longer time scale than do the processes in the parallel direction, which leads to the setup of the source sink equilibrium. We thus describe this "fluctuation" dynamic separately, as is also the case traditionally. Starting from noise, the most unstable mode develops. Depending on parameters the mode bursts into turbulence, which leads to anomalous radial transport, which in turn leads to a flattening of the density profile. The parallel velocities show a small and nearly linear increase toward ion sound speed at the sheath entry, while the plasma potential becomes positive toward the plasma axis, as is expected from a neutral plasma source and initial electron losses into the sheath. The temporal development of the total perpendicular kinetic energy
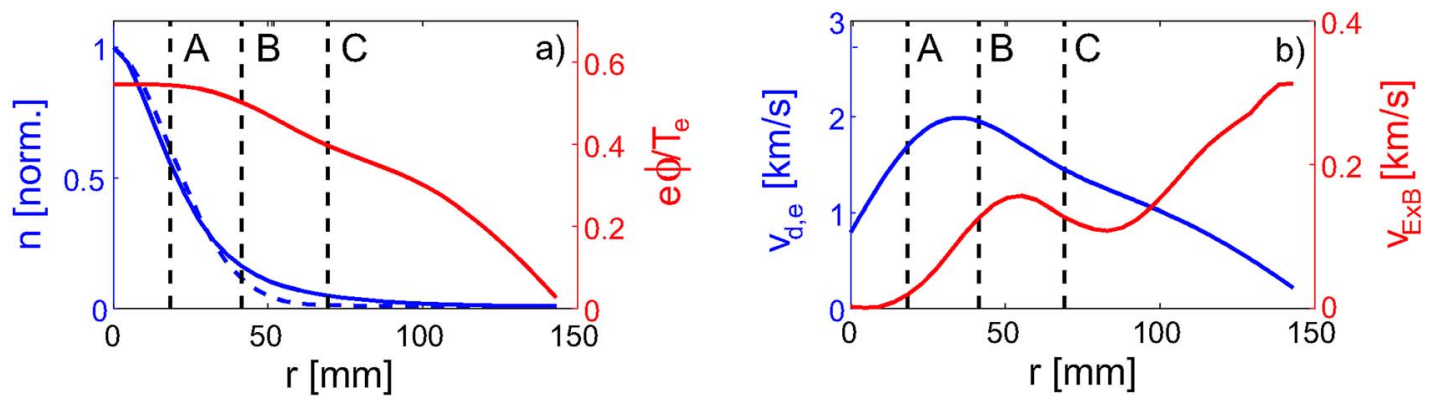

FIG. 7. (Color online) Time averaged radial profiles of density and potential (left), with the dashed line indicating the source profile. On the right side, the diamagnetic and $E \times B$ velocities. All data taken at $z=L_{z} / 2$. The three positions indicated are the maximum gradient region, the foot of the plasma and the far edge, from inside to outside. 


\section{linear}

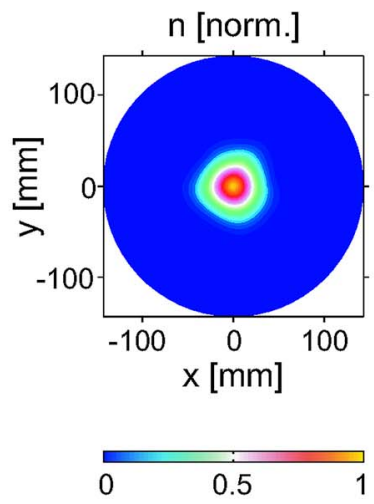

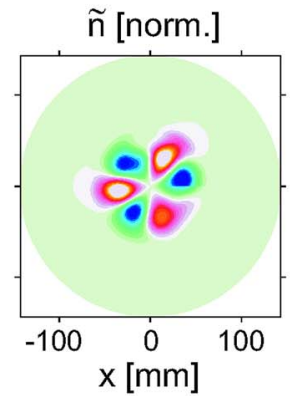

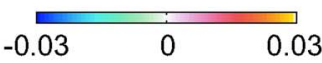

\section{$\underline{\text { nonlinear }}$}
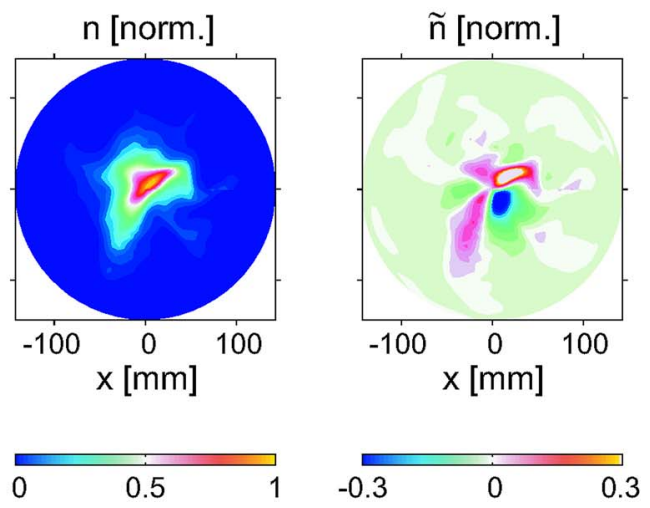

FIG. 8. (Color online) Plasma density and its fluctuations in the radial-azimuthal $(r, \theta)$ plane for the linear (left) and nonlinear phase (right). The time instants are indicated in Fig. 5 as the left and right side bars, respectively.

$$
E_{\perp, t o t}=\int\left(n \nabla_{\perp} \phi\right)^{2} d^{3} r
$$

is shown in Fig. 5. The energy in the linear phase is characterized by an exponential growth until $t \geq 2 \mathrm{~ms}$ and subsequent nonlinear saturation in the developed turbulent state. In agreement with the linear dispersion properties of the model, the mode number $\widetilde{m}$ of the linear mode destabilized depends on the circumference measured in $\rho_{s}$ of the plasma column at the location of the maximum density gradient. This is depicted in Fig. 6 for $\nu_{i}=0.075$ and varying values of the source width $L_{n}$, a feature that corresponds roughly to increasing the magnetic field strength in experiments. The typical structure of the density and potential profiles are shown in Fig. 7 together with the diamagnetic and $E \times B$ velocities, showing the convex feature of the $E \times B$ velocity that develops in response to the sheath boundary conditions.

The corresponding spatiotemporal structure of the plasma density in the radial-azimuthal $(r, \theta)$ plane is depicted for the linear and nonlinear phases in Fig. 8 (the time instants are indicated in Fig. 5 by the two vertical lines). Shown are both the plasma density and its fluctuation part, which is obtained by subtracting the azimuthally averaged density. Note that this is in contrast to experimental procedures, where the time average is subtracted to obtain the fluctuations. In the linear phase, the radial density profile resembles very much the initial profile and only a slight broadening of the profile due to cross-field transport is observed. The density fluctuations reveal that a coherent drift wave mode with mode number $\widetilde{m}=3$ is present in this linear phase. The mode propagates purely azimuthally with electron diamagnetic drift, in agreement with the linear dispersion relation. In contrast to the linear phase, in the nonlinear saturated phase the radial density profile is strongly broadened and perturbed due to strong radial fluctuation-induced transport. The turbulent fluctuations are so strong that they dominate the spatiotemporal structure of the density profile, showing that the assumption of small fluctuations on top of a background profile clearly does not apply to this situation. However, in the maximum radial plasma density gradient region still some quasicoherent mode structure with small mode numbers of typically $\tilde{m} \leq 3$ is observed. This general feature of the turbulent state is more pronounced in the time-averaged mode number spectrum, shown in Fig. 9(a). The spectrum is dominated by quasicoherent modes with mode numbers $\tilde{m}=1-3$. Using wavelet analysis, the temporal evolution of the mode number spectrum is obtained [Fig. 9(b)]. The dominance of the low mode numbers is evident. They are not strictly coherent, but exist only transiently. This behavior is in good agreement with experimental observations, in which it was found that the energy is transferred between low mode number drift wave modes in weakly developed turbulence using bicoherence analysis. ${ }^{27}$ The increased fluctuation-induced transport in the turbulent state leads to the formation of density events, which are not only observed in the gradient region, but also in the radially far plasma edge, where the time-averaged background density is small. The time series a)

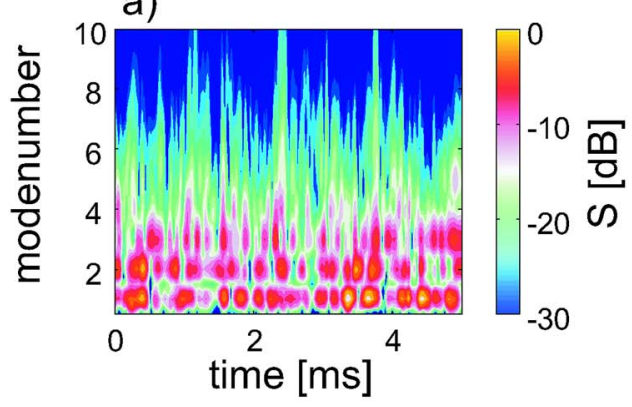

b)

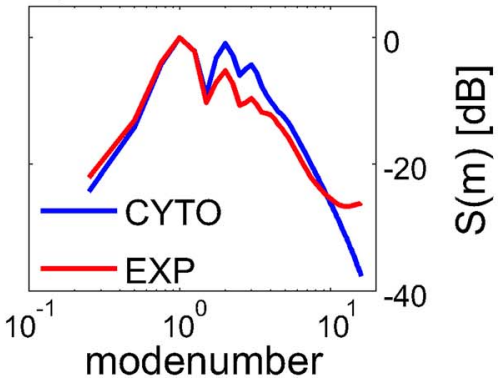

FIG. 9. (Color online) Temporal evolution of the mode number spectrum (a) and its time average (b) over a time interval of $5 \mathrm{~ms}$. Both spectra are evaluated in the maximum radial plasma density region at $r=30 \mathrm{~mm}$. Note that $t=0$ corresponds to the blue vertical line in Fig. 5. For the timeaveraged spectrum, results from simulation and experiment are shown. 
Pos.
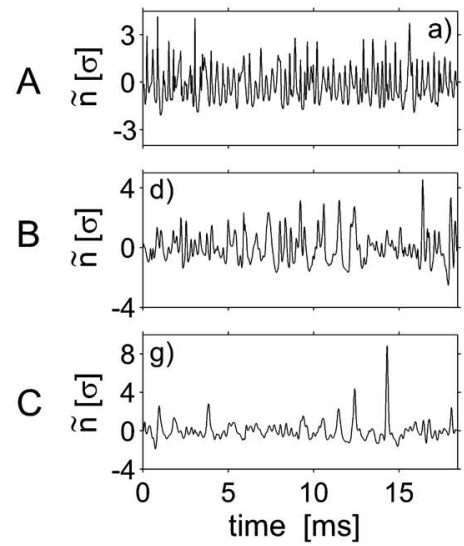

of density fluctuations together with the corresponding probability density functions (PDFs) and frequency spectra are shown in Fig. 10 for the maximum radial gradient region, a position at the foot of the density gradient and the far plasma edge, as indicated in Fig. 7. In the gradient region, the contribution of the quasicoherent fluctuations is observed, which lead to a peak in the frequency spectrum at a frequency of $f=3 \mathrm{kHz}$. The PDF is broad and has a slightly enhanced positive tail with skewness $s=0.75$ and kurtosis $K=0.45$ in this region where the fluctuations originate. The intermittency of the fluctuations becomes much more pronounced toward the plasma edge. There, the density PDF is strongly skewed toward large positive plasma density fluctuation events, which appear in the time series as sporadic distinct density bursts with large relative fluctuation amplitude up to $8 \sigma$. Consequently, the skewness and kurtosis increase to $s$ $=0.86$ and $K=1.17$ at the foot of the density gradient (position B) to reach $s=3.4$ and $K=21$ for the far edge (position C). In the gradient region the frequency spectrum shows a pronounced feature at $f=3.2 \mathrm{kHz}$, which is ascribed to the quasicoherent mode observed, with a decay for high frequencies following a power law $S \sim f^{-\alpha}$ with $\alpha=4$.6. In the far

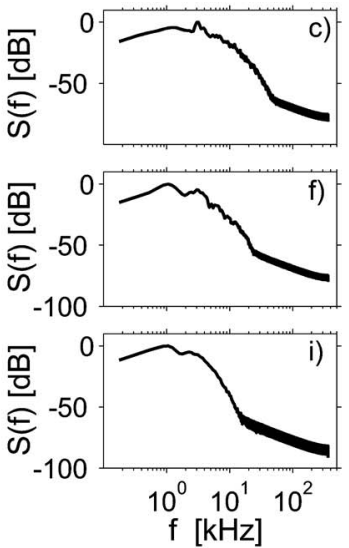

FIG. 10. Time series of density fluctuations $\widetilde{n}$ normalized to standard deviation (left column), the corresponding PDFs (middle column) and frequency spectra (right column) for three different radial positions, as indicated in Fig. 7. The top row corresponds to the maximum radial density gradient region at $r=20 \mathrm{~mm}$; the bottom row to the far plasma edge at $r$ $=75 \mathrm{~mm}$.

edge, the frequency spectrum shows no pronounced peak and rolls off to a similar power law behavior as in the gradient region. The lack of a pronounced peak, reflecting the time between bursts of about a millisecond $(f=1 \mathrm{kHz})$, as is observed at position $\mathrm{B}$ at the foot of the gradient, reflects the weakening contribution of these events to the total spectral energy with increasing radius. The amplitude of the large density bursts is comparable to the amplitude of fluctuations in the gradient region, showing that they directly convect density from the gradient region into the far plasma edge. The spatiotemporal dynamics of density fluctuations are plotted time resolved in Fig. 11, which shows the density fluctuations and overlayed the contours of the potential, which are the streamlines of the $E \times B$ velocity. The potential structures extend radially far, providing a large radial region of correlated $E \times B$ velocity, which is able to convect plasma density outwards. The overall dynamics of the system reveals features similar to the ones found in the tokamak SOL, where the occurrence of plasma blobs is agreed to be responsible for the observed fluctuation statistics and numerical simulations have shown the importance of background turbulence interaction as well. ${ }^{28,29}$ It should, however, be noted
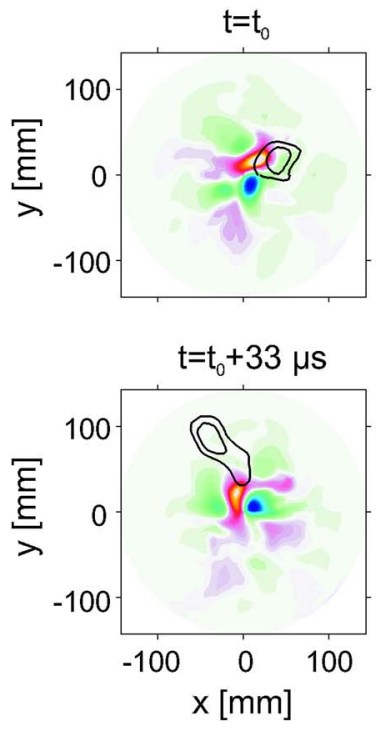
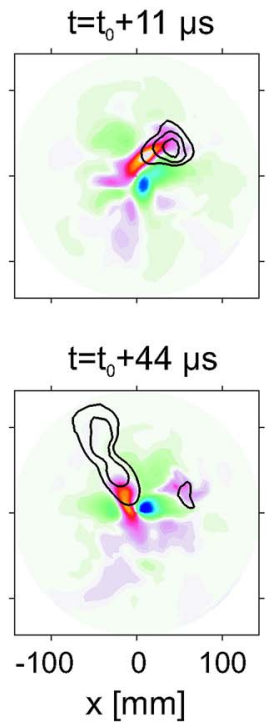

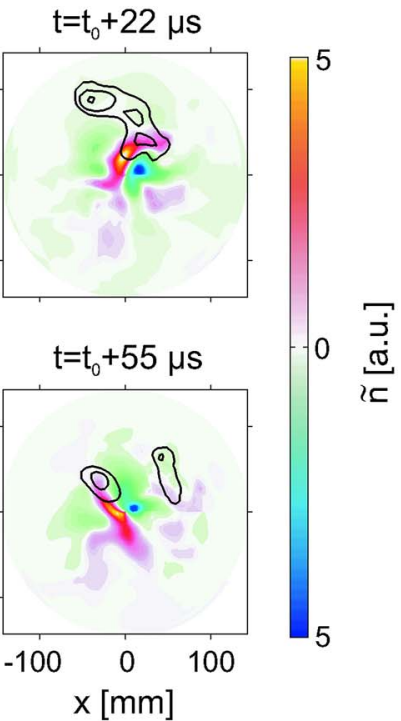

FIG. 11. (Color online) Temporally resolved color-coded plots of density fluctuations in the azimuthal-radial cross section. Fluctuations of the electric potential are superimposed as contour lines. 
that in the tokamak SOL the magnetic field geometry is made responsible for the radial acceleration and far propagation of the structures, ${ }^{30}$ a mechanism that is clearly absent here. The presented numerical simulations will allow to investigate the formation of the intermittent transport observed in good detail. Investigations on this topic are ongoing and results will be reported in a dedicated paper.

\section{CONCLUSION}

We presented the fluid equations determining the full dynamical evolution of background and fluctuations in a magnetized cylindrical plasma experiment. The corresponding linear dispersion relation was derived and the full set of nonlinear equations solved numerically with appropriate boundary conditions. The 3D simulations show good agreement with the phenomenology of the experimentally obtained data and thus prove useful to augment the diagnostics of such experiments. Thus, for the first time, turbulence simulations of a full plasma device have been performed in which the plasma profile developed self-consistently. This provides a testing ground for the validity of the plasma fluid description in a bounded plasma system. More results on specific issues, such as blob formation and increasing intermittency with radial position, will be published later. The code for the simulations is available from the authors on request.

${ }^{1}$ T. Klinger, A. Latten, A. Piel, and G. Bonhomme, Plasma Phys. Controlled Fusion 39, B145 (1997).

${ }^{2}$ T. Pierre, G. Leclert, and F. Braun, Rev. Sci. Instrum. 58, 6 (1987).

${ }^{3}$ W. Gekelman, H. Pfister, Z. Lucky, J. Bamber, D. Leneman, and J. Maggs, Rev. Sci. Instrum. 62, 2875 (1991).

${ }^{4}$ E. Wallace, E. Thomas, A. Eadon, and J. Jackson, Rev. Sci. Instrum. 75, 5160 (2004).

${ }^{5}$ O. F. Castellanos, E. Anabitarte, J. M. Sentyes, C. Hidalgo, and M. A. Pedrosa, Plasma Phys. Controlled Fusion 47, 2067 (2005).

${ }^{6}$ C. Holland, G. R. Tynan, J. H. Y. A. James, D. Nishijima, M. Shimada, and N. Taheri, Plasma Phys. Controlled Fusion 49, A109 (2007).

${ }^{7}$ G. R. Tynan, M. Burin, C. Holland, G. Antar, and N. Crocker, Phys. Plasmas 11, 5195 (2004).

${ }^{8}$ C. M. Franck, O. Grulke, and T. Klinger, Phys. Plasmas 9, 3254 (2002).

${ }^{9}$ V. Naulin, J. Nucl. Mater. 363-365, 24 (2007).

${ }^{10}$ A. Hasegawa and M. Wakatani, Phys. Rev. Lett. 50, 682 (1983).

${ }^{11}$ R. F. Ellis, E. Marden-Marshall, and R. Majeski, Plasma Phys. 22, 113 (1980).

${ }^{12}$ P. P. Sosenko, G. Bonhomme, and T. Pierre, J. Plasma Phys. 63, 157 (2000).

${ }^{13}$ C. Schröder, O. Grulke, T. Klinger, and V. Naulin, Phys. Plasmas 9, 4249 (2004).

${ }^{14}$ C. Schröder, T. Klinger, D. Block, A. Piel, G. Bonhomme, and V. Naulin, Phys. Rev. Lett. 86, 5711 (2001).

${ }^{15}$ V. Naulin, J. Nycander, and J. Juul Rasmussen, Phys. Rev. Lett. 81, 4148 (1998).

${ }^{16}$ V. Naulin, O. E. Garcia, A. H. Nielsen, and J. Juul Rasmussen, Phys. Lett. A 321, 355 (2004).

${ }^{17}$ R. F. Ellis and R. W. Motley, Phys. Fluids 17, 583 (1974).

${ }^{18}$ N. Kasuya, M. Yagi, and K. Itoh, J. Plasma Phys. 72, 957 (2006).

${ }^{19}$ M. Wakatani, K. Watanabe, H. Sugama, and A. Hasegawa, Phys. Fluids B 4, 1754 (1992).

${ }^{20}$ C. Schröder, O. Grulke, T. Klinger, and V. Naulin, Phys. Plasmas 12, 042103 (2005)

${ }^{21}$ P. C. Stangeby, The Plasma Boundary of Magnetic Fusion Devices (Institute of Physics, Bristol, 2000).

${ }^{22}$ D. B. Heifetz, Physics of Plasma-Wall Interactions in Controlled Fusion, edited by D. E. Post and R. Behrisch, NATO Advanced Studies Institute Series (Plenum, New York, 1984), Vol. 131.

${ }^{23}$ A. Arakawa, J. Comput. Phys. 1, 119 (1966).

${ }^{24}$ G. E. Karniadakis, M. Israeli, and S. A. Orszag, J. Comput. Phys. 97, 414 (1991).

${ }^{25}$ V. Naulin and A. H. Nielsen, SIAM J. Sci. Comput. (USA) 25, 104 (2003).

${ }^{26}$ A. Latten, Ph.D. thesis, Christian-Albrechts-Universität, Kiel, Germany (1997).

${ }^{27}$ F. Brochard, T. Windisch, O. Grulke, and T. Klinger, Phys. Plasmas 13, 122305 (2006)

${ }^{28}$ O. E. Garcia, V. Naulin, A. H. Nielsen, and J. Juul Rasmussen, Phys. Rev. Lett. 92, 165003 (2004).

${ }^{29}$ O. E. Garcia, V. Naulin, A. H. Nielsen, and J. Juul Rasmussen, Phys. Plasmas 12, 062309 (2005).

${ }^{30}$ O. E. Garcia, N. H. Bian, V. Naulin, A. H. Nielsen, and J. Juul Rasmussen, Phys. Plasmas 12, 090701 (2005). 